\title{
56. Differential Chromosomal Characteristics in the Funa Subspecies (Carassius)
}

\author{
By Takayoshi UEDA and Yoshio OJIMA \\ Department of Biology, Faculty of Science, \\ Kwansei Gakuin University, Nishinomiya \\ (Communicated by Sajiro Makino, M. J. A., June 15, 1978)
}

The Funa (Carassius auratus) is a common species of the fresh water teleosts of the Cyprinidae having a world-wide distribution. There is, however, a considerable discrepancy on its systematic classification, because of its polymorphic distributional features. Nakamura (1969) divided the Japanese genus Carassius into the following five subspecies: C. a. subsp.; C. a. langsdorfii; C. a. grandoculis; C. a. buergeri; and C.a.cuvieri. This is a unique classification-system in this genus. The chromosomes of $C$. a. cuvieri have been reported as $2 n, 100$ in both sexes (Ojima et al. 1966, 1967). Kobayashi et al. (1970, 1973) reported the chromosome number of $C$. $a$. subsp. from Lake Kasumigaura as $2 n, 100$ in both sexes. According to them, C. $a$. langsdorfi from Lake Shinji had $2 n, 100$ in both male and female specimens. C.a.langsdorfii from Miyazaki district is in the same condition in their sexuality and chromosome number. In contrast, there were only female specimens having 3 n-range and 4n-range chromosome numbers in those from the Kanto district (Kobayashi et al. 1970). It was remarked that 3 -individuals of $C$. a. langsdorfii propagated unisexually by gynogenesis (Kobayashi et al. 1970, 1972). Ojima et al. (1977) showed that the population of C.a.langsdorfii occurring in Lake Biwa was mostly consisted of females with 3nchromosomes which bred by gynogenesis. A lot of similarities can be found between C. a. buergeri and C. a. langsdorfii in their sexuality and chromosome numbers. C. a. buergeri from Lake Suwa showed $50: 50$ sex ratio and the chromosome number of $2 n, 100$ in both sexes. The Hokkaido population consisted, however, of females which were characterized by 3 n-chromosomes as well as by gynogenetic reproduction (Kobayashi et al. 1973).

Impressive is the fact that the short arms of the large submetacentrics of the 3n-range complement of C. a. langsdorfii occurring in Lake Biwa were intensely C-banded. The present report deals with some C-banded markers found in 5 subspecies of Carassius after the Nakamura's classification-system (1969).

Materials and methods. Five females of C. a. langsdorfii from 
Lake Biwa; 3 males and 2 females of $C$. a. cuvieri from Lake Biwa; 4 males and 2 females of $C$. a. grandoculis from Lake Biwa; 2 males and 2 females of $C$. a. buergeri from Lake Suwa; 5 females of $C . a$. subsp. from Lake Kasumigaura, and 4 males and 2 females from Pond Jyo (Gunma) provided materials for study. Their subspecific identification followed the descriptions of Nakamura (1969). The kidney culture method was applied to all of them together with ovary cultures for females. In some cases, the direct chromosome preparations from the kidney tissue were made with satisfactory results. The Giemsa C-banding technique was applied to them for the chromosome analysis.

Results and discussion. Fig. 1 is the karyotype of C. a. langsdorfii from Lake Biwa, which is characterized by a pair of marker chromosomes with deeply stained C-bands on the short arms of the second largest submetacentric chromosomes. The karyotype of C. $a$. cuvieri (Fig. 2) showed the chromosome number of $2 n, 100$ in both sexes, which consisted of 6 pairs of metacentric chromosomes, 18 pairs of submetacentric chromosomes and 26 pairs of acro- or telocentric chromosomes, as shown by Ojima et al. $(1966,1967)$. Many deeply stained C-bands were shown in the centromere regions. The markers as found in C.a.langsdorfii could not be demonstrated in this subspecies. Generally speaking, C. a. cuvieri showed no difference in the C-band pattern between males and females. Figs. 3 and 4 show the karyotypes of C.a.grandoculis and C.a.buergeri, respectively, which indicate the chromosome number of $2 n, 100$ with the same constitution as that of C. a. cuvieri. The same is with the C-band

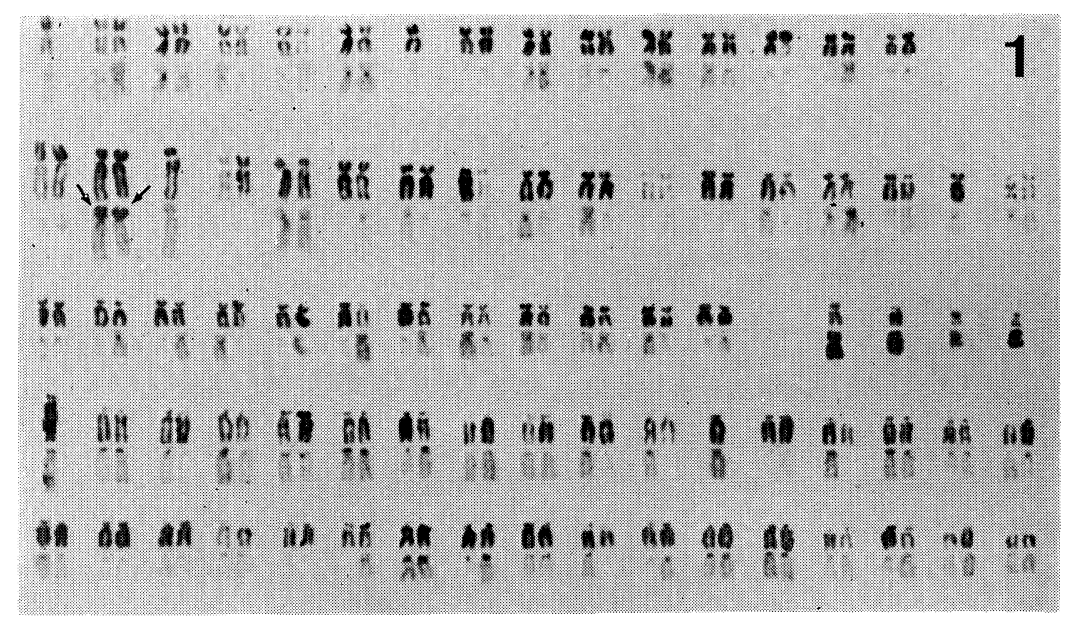

Fig. 1. C-banding karyotype of C.a.langsdorfii (the lower row). The arrows denote two large submetacentrics having intensely C-banded short arms. 
pattern; as in C.a. cuvieri many deeply stained bands were found in the centromere regions. No remarkable sexual difference of the karyotype was detected. In Figs. 5 and 6 are presented the karyotypes of females and males of $C$. $a$. subsp. They were characterized by $2 n, 100$ as well as by the same constitution as that of $C$. a. cuvieri. In contrast, the C-band pattern was corresponding to that of C.a.langsdorfi: there were markers with C-banded short arms of the second largest submetacentrics. Noticeable is the occurrence of two markers in the female while one in the male.



Fig. 2. C-banding karyotype of C. a. cuvieri (the lower row).

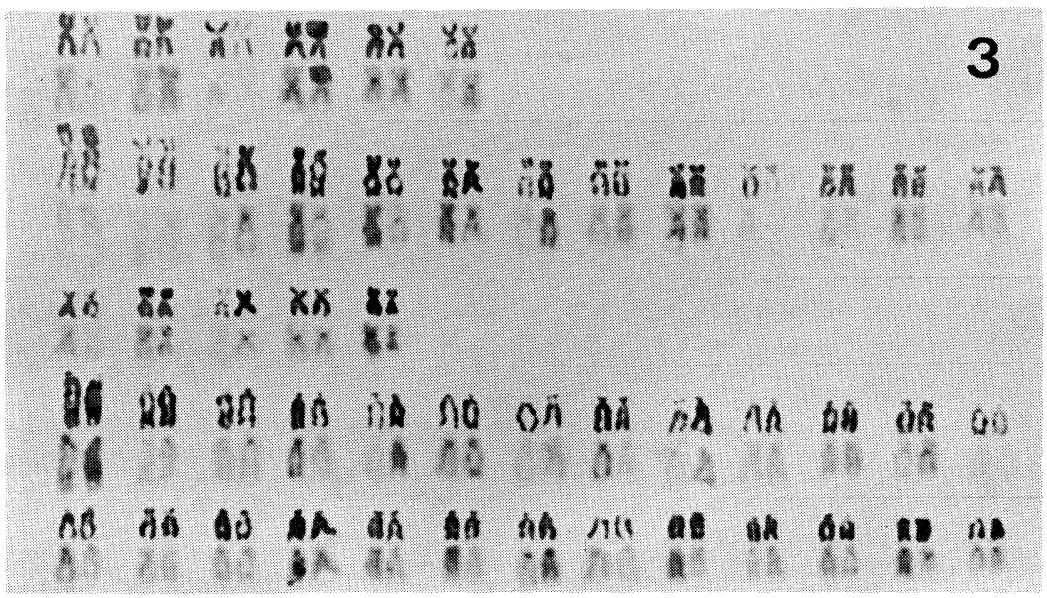

Fig. 3. C-banding karyotype of C.a.grandoculis (the lower row).

In some teleost species the existence of possible heteromorphic sex chromosomes has been reported by several investigators. The situation seems to deal with the sexual difference of chromosomes in teleost fishes. However, the literature refers to no such picture 


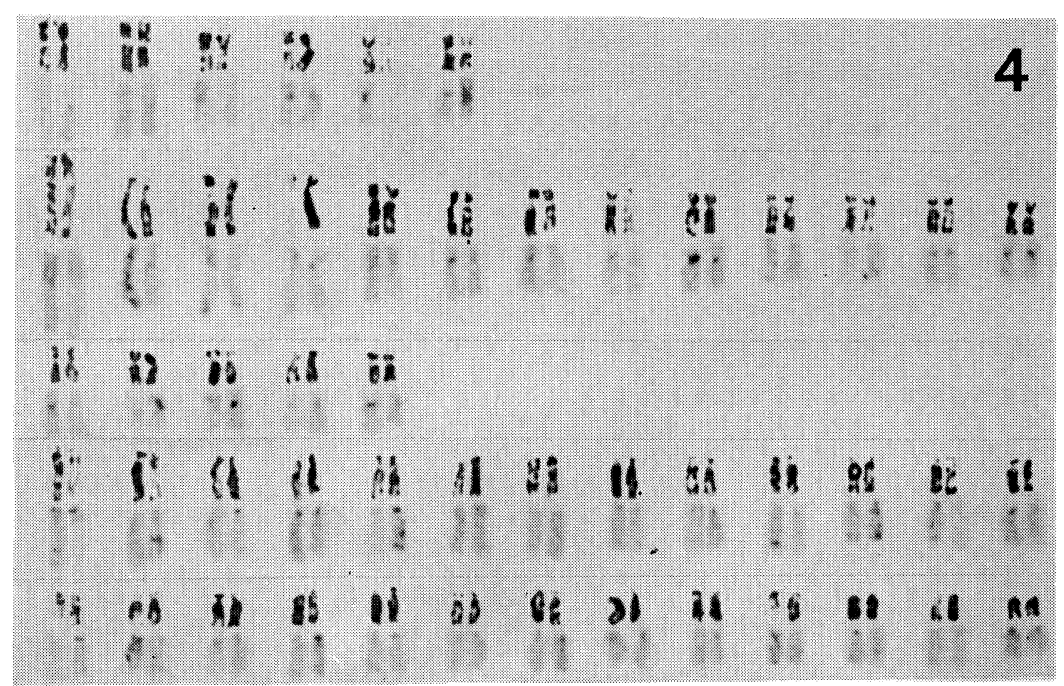

Fig. 4. C-banding karyotype of C. a. buergeri (the lower row).

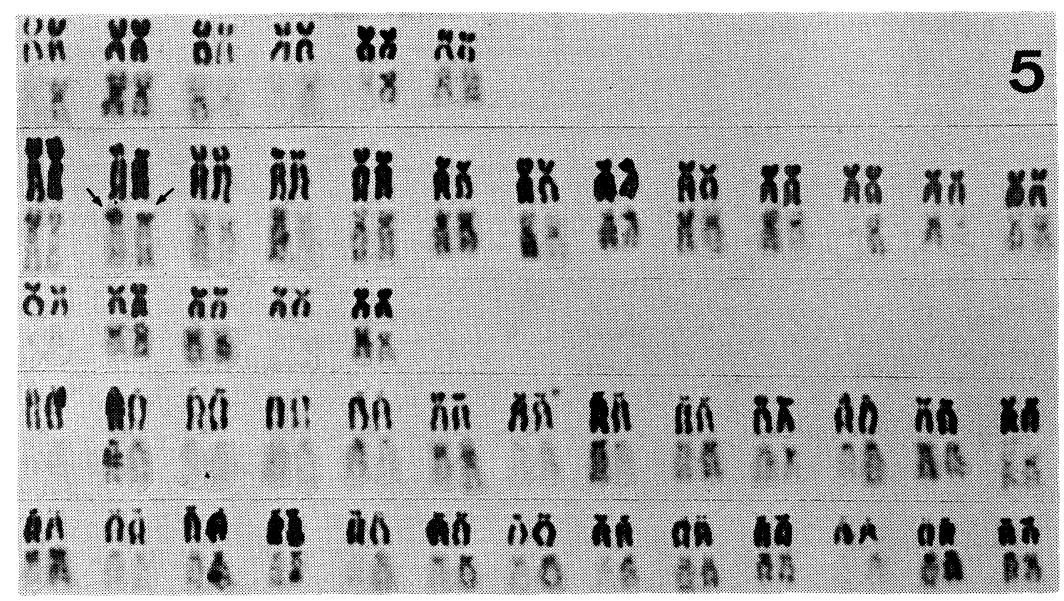

Fig. 5. C-banding karyotype of a female of C.a.subsp. (the lower row). The arrows denote two large submetacentrics having intensely C-banded short arms.

in the larger part of teleosts. For understanding of sex determination in teleosts the following items of elucidation would be required: 1) the cytogenetic mechanism, 2) morphophysiological process, and 3) hormonal control. In teleosts, our knowledge is very poor on these three aspects in comparison with other animals (Vanyakia 1972). Svärdson and Wickbom (1942) have mentioned that the cytological identification of the sex chromosomes in teleosts is impossible even though the existence of such features were proven by genetic methods. Makino (1932) and Galgano (1938) are of opinion that inasmuch as 
teleosts occupy a rather low position on the evolutionary ladden, the degree of difference between their sex chromosomes and autosomes being very negligible. On the basis of a consensus of estimates through the facts and views as mentioned above, the statements may be available that the fish group is now being in the course of evolution, and that the sexual differentiation is still in unstable condition.

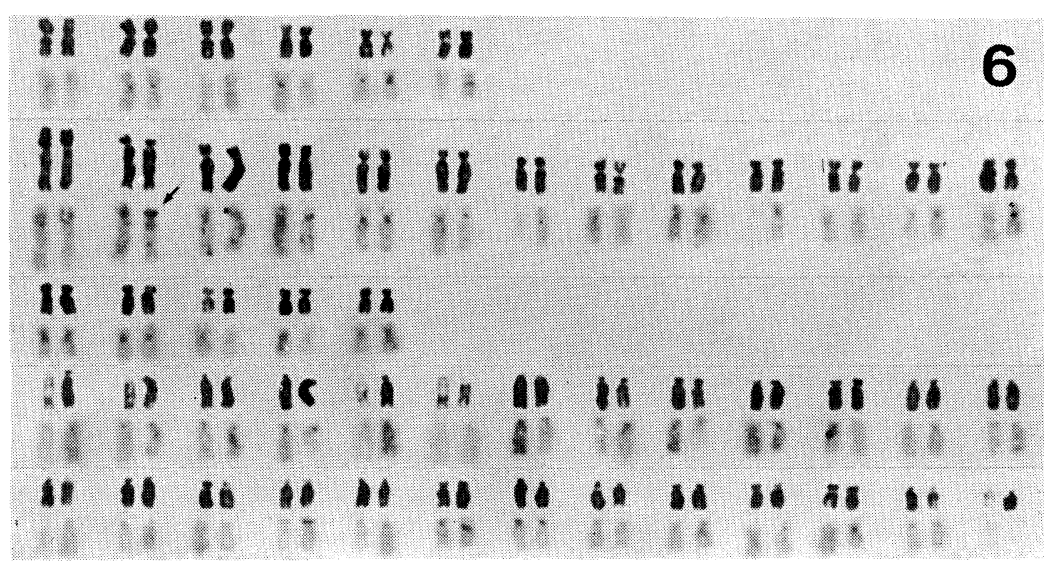

Fig. 6. C-banding karyotype of a male of C. $a$. subsp. (the lower row). The arrow denotes one large submetacentrics having intensely C-banded short arms.

According to Gosline (1971), the teleostean fishes are classified into the following three groups: lower (Salmoniformes, Cypriniformes, etc.), intermediate (Cyprinodontiformes, etc.), and higher groups (Perciformes, etc.). According to this view, the Cyprinidae belongs to the lower group. The fact showing that the sexual difference for the C-banded marker chromosomes occurs in certain subspecies of Carassius would be suggestive of sex differentiation in the lower teleostean group.

Summary. In five subspecies of Carassius auratus, such as $C . a$. langsdorfi, C. a. cuvieri, C. a. grandoculis, C. a. buergeri, and C. a. subsp., the C-banding patterns of chromosomes are comparatively investigated. C. a. langsdorfii is characterized by a pair of markers with remarkable C-bands on the short arms of the second largest submetacentrics. In $C$. $a$. subsp. there are two such markers in the female and one in the male.

Our particular thanks are due to Emeritus Professor Sajiro Makino, M. J. A., for his invaluable advice and revision of the manuscripts. 


\section{References}

Galgano, H. (1938): Atti Soc. Ital. Anat., no. 7.

Gosline, W. A. (1971): Functional Morphology and Classification of Teleostean Fishes. Univ. Press Hawaii, Honolulu, pp. 1-208.

Kobayashi, H., Y. Kawashima, and N. Takeuchi (1970): Jap. J. Ichthyol., 17, 153-160.

Kobayashi, H., and N. Ochi (1972): Zool. Mag. (Tokyo), 81, 67-71.

Kobayashi, H., H. Ochi, and N. Takeuchi (1973) : Jap. J. Ichthyol., 20, 7-12.

Makino, S. (1932) : Proc. Imp. Acad. Japan, 8, 20-26.

Nakamura, M. (1969): Cont. from the Res. Inst. for National Resources, no. 1198, pp. 266-282.

Ojima, Y., S. Hitotsumachi, and S. Makino (1966) : Proc. Japan Acad., 42, 62-66.

Ojima, Y., and S. Hitotsumachi (1967): Jap. J. Genetics, 42, 163-167.

Ojima, Y., and N. Asano (1977) : Proc. Japan Acad., 53B, 138-142.

Svärdson, G., and T. Wickbom (1942) : Hereditas, 28, nos. 1-2.

Vanyakia, E. D. (1972) : Genetics, Selection, and Hybridization of Fish (Cherfas,

B. I. ed.), Israel Program for Scientific Translations Ltd., Jerusalem, pp. 5-24. 